\title{
Selective assessment of duplex heat-treated wood by near-infrared spectroscopy with principal component and kinetic analyses
}

\author{
Tetsuya Inagaki $^{1} \cdot$ Yuuki Asanuma $^{1} \cdot$ Satoru Tsuchikawa $^{1}$
}

Received: 21 July 2017 / Accepted: 25 September 2017 / Published online: 2 November 2017

(C) The Japan Wood Research Society 2017

\begin{abstract}
We selectively assessed the thermal and hygrothermal treatment times of duplex heat-treated samples from the softwood hinoki cypress (Chamaecyparis obtusa) and the hardwood Japanese zelkova (Zelkova serrata) using near-infrared (NIR) spectroscopy with principal component analysis (PCA) and spectral-kinetic analysis. Wood samples from each species were thermally or hygrothermally treated at $120,130,150$, and $180{ }^{\circ} \mathrm{C}$, and the secondderivative spectra of these samples in the $6300-5450 \mathrm{~cm}^{-1}$ range, where moisture content has the smallest effect, were then subjected to PCA. The master curve that was calculated by kinetic analysis successfully explained changes in the first principal component (PC1) scores with thermal treatment time for all temperatures. The angles between the PC1 loadings that explained the spectral variation due to thermal and hygrothermal treatment were $79^{\circ}$ for hinoki and $80^{\circ}$ for zelkova. Thus, calculation of the inner product between the second-derivative spectra of duplex heat-treated wood and a loading vector that explained the spectral variation due to thermal or hygrothermal treatment allowed us to selectively assess the thermal and hygrothermal treatment times.
\end{abstract}

Keywords NIR spectroscopy · Kinetic analysis · TTSP method $\cdot$ Duplex thermally treated wood $\cdot$ Selective assessment

Tetsuya Inagaki

inatetsu@agr.nagoya-u.ac.jp

1 Graduate School of Bioagricultural Sciences, Nagoya University, Furo-cho, Chikusa-ku, Nagoya 464-8601, Japan

\section{Introduction}

Heat treatment confers several advantages on wood, such as improving its strength, dimensional stability, resistance to fungal decay, and color. There are many types of thermal modification, which vary according to temperature, atmosphere, and duration [1]. The atmosphere in which the treatment occurs (i.e., in air, under a vacuum, in steam, or in water) has a significant effect on the chemical structure of the wood. In general, treatments that occur under oxidizing conditions (hereafter referred to as "thermal treatments", this treatment is also called as "dry" thermal treatment) result in an increase in carbonyl-containing species and a decrease in $\mathrm{OH}$ groups, while treatments in steam (hereafter referred to as "hygrothermal treatments", this treatment is also called as "wet" thermal treatment) result in the accelerated formation of organic acids that catalyze the hydrolysis of hemicellulose and to a lesser extent amorphous cellulose.

A non-destructive method is required to determine the chemical components of wood that is being heat-treated for industrial purposes. Near-infrared (NIR) spectroscopy has been reported to have high application potential as a nondestructive tool for the rapid determination of the chemical, mechanical, and physical properties of wood in the wood products industry [2,3]. However, the complexity of the NIR spectra of wood makes the use of this tool problematic, as the broad absorption bands of this complex polymer and the influence of the physical condition of a sample can make the spectra difficult to analyze. Multivariate statistical methods that are based on the projection of spectral information to latent structures, such as principal component analysis (PCA) and partial least squares (PLS) regression, are promising for the quantitative and qualitative analysis of such complex data. However, to prepare a robust calibration curve, the training sample set needs to contain a sufficient 
range of the properties under evaluation and to represent all of the important sources of variability that would be included in the samples that will be predicted, including the wood species, moisture content, density, and surface roughness, meaning that a large sample size is required.

We previously proposed a new method for determining the properties of heat-treated wood samples that combined unsupervised spectral analysis and kinetic analysis [4]. This method used PCA with the aid of kinetic analysis to understand changes in the NIR spectra of heat-treated wood under dry conditions and showed that kinetic analysis successfully explained the changes in the second-derivative spectral bands and the PCA scores calculated from the second-derivative NIR spectra of heat-treated sugi (Cryptomeria japonica D. Don) samples at temperatures of $90,120,150$, and $180^{\circ} \mathrm{C}$. Therefore, in the present study, we used this method to selectively assess the thermal and hygrothermal treatment times from the NIR spectra of the softwood hinoki cypress [Chamaecyparis obtusa (Siebold \& Zucc.) Endl.] and the hardwood Japanese zelkova [Zelkova serrata (Thun.)].

PCA is a linear method that allows multi-dimensional data to be projected onto a few orthogonal features called principal components (PCs). Therefore, if the calculated loading vectors, which represent the direction of spectral variation in the dataset, from thermally treated and hygrothermally treated wood spectra are orthogonal, PCA should be able to selectively assess the effects of thermal and hygrothermal treatment from the NIR spectra of duplex heat-treated wood. To analyze this, we prepared thermally or hygrothermally treated softwood and hardwood samples ("single treatment") at various temperatures and over a range of treatment times and measured their NIR spectra. We then treated the thermally treated wood samples with an additional hygrothermal treatment and treated the hygrothermally treated samples with an additional thermal treatment to produce duplex-treated wood samples and again measured their NIR spectra. The NIR spectra of the single-treated wood samples demonstrated that kinetic analysis is useful for understanding changes in the PCA scores calculated from NIR spectra of wood subjected to thermal and hygrothermal treatments. Furthermore, we found that this method can also be used to selectively assess the effects of thermal and hygrothermal treatments from the NIR spectra of duplextreated wood using the loading vectors calculated from the NIR spectra of single-treated wood. It is expected that this method will be suitable for the analysis of archaeological wood samples, as the inclusion of kinetic analysis allows score changes to be predicted at specific temperatures.

\section{Materials and methods}

\section{Samples and treatments}

Hinoki cypress and Japanese zelkova wood samples were each cut from the same board containing the same tree ring structure. The sample dimensions were $20 \mathrm{~mm}$ (tangential direction) $\times 20 \mathrm{~mm}$ (radial direction) $\times 50 \mathrm{~mm}$ (longitudinal direction), where the direction of the wood fiber was considered to be parallel to the longitudinal direction of the sample wherever possible. Three of the samples from each species were thermally treated at $120,130,150$, and $180^{\circ} \mathrm{C}$ for the periods summarized in Table 1, while two additional samples were hygrothermally treated. After the temperature in electric oven (EYELA, NDO400; natural convection oven) reached the target temperature, the wood samples were put into oven for thermal treatment. Hygrothermal treatment was done by placing wood samples in a high-pressure cracking reactor (SAN-AI Kagaku Co.Ltd., HU-100: composed of PTFE inner cylinder and stainless steel external cylinder. The internal volume is about $240 \mathrm{~cm}^{3}$ ) with a piece of wet paper and putting them into an electric oven after the temperature in oven reached the target temperature.

Furthermore, to investigate the possibility of selective assessment of thermal treatment and hygrothermal treatment, we prepared 24 kinds of duplex heat-treated wood samples, as summarized in Table 2. The detail description about the duplex heat treatment is explained in chapter "Selective assessment of duplex heat-treated wood" in results and discussion.

Table 1 Thermal treatment period

\begin{tabular}{lllllllrrr}
\hline & Temp. $\left({ }^{\circ} \mathrm{C}\right)$ & Time $(\mathrm{h})$ & Time $(\mathrm{h})$ & Time $(\mathrm{h})$ & Time $(\mathrm{h})$ & Time $(\mathrm{h})$ & Time $(\mathrm{h})$ & Time $(\mathrm{h})$ & Time $(\mathrm{h})$ \\
\hline Thermal treatment & 120 & 96 & 192 & 384 & 768 & 1152 & 1536 & 3072 & 6144 \\
& 130 & 24 & 48 & 96 & 192 & 288 & 384 & 768 & 1536 \\
& 150 & 6 & 12 & 24 & 48 & 72 & 96 & 192 & 384 \\
Hygrothermal treatment & 180 & 0.875 & 1.75 & 3.5 & 7 & 10.5 & 14 & 28 & 56 \\
& 120 & 14 & 27 & 55 & 110 & 166 & 219 & 439 & 878 \\
& 130 & 3 & 7 & 14 & 27 & 41 & 55 & 110 & 219 \\
& 150 & 1 & 2 & 3 & 7 & 10 & 14 & 28 & 55 \\
& 180 & 0.1 & 0.25 & 0.5 & 1 & 1.5 & 2 & 4 & 8 \\
\hline
\end{tabular}


Table 2 Condition for duplex thermal treatment

\begin{tabular}{llrlll}
\hline & \multicolumn{2}{l}{ Thermal } & & \multicolumn{2}{l}{ Hygrothermal } \\
\cline { 2 - 3 } \cline { 5 - 6 } & Temp. $\left({ }^{\circ} \mathrm{C}\right)$ & Time $(\mathrm{h})$ & & Temp. $\left({ }^{\circ} \mathrm{C}\right)$ & Time $(\mathrm{h})$ \\
\hline 1 & 120 & 96 & & 180 & 3 \\
2 & 120 & 768 & & 180 & 3 \\
3 & 120 & 6144 & & 180 & 8 \\
4 & 130 & 24 & & 180 & 8 \\
5 & 130 & 192 & & 180 & 6 \\
6 & 130 & 1536 & & 180 & 8 \\
7 & 150 & 6 & & 180 & 6 \\
8 & 150 & 48 & & 180 & 3 \\
9 & 150 & 384 & 180 & 6 \\
10 & 180 & 1 & 180 & 6 \\
11 & 180 & 7 & 180 & 3 \\
12 & 180 & 56 & 180 & 8 \\
\hline
\end{tabular}

\begin{tabular}{llllll}
\hline & \multicolumn{2}{l}{ Hygrothermal } & & & \multicolumn{2}{l}{ Thermal } \\
\cline { 5 - 6 } & Temp. $\left({ }^{\circ} \mathrm{C}\right)$ & Time $(\mathrm{h})$ & & Temp. $\left({ }^{\circ} \mathrm{C}\right)$ & Time $(\mathrm{h})$ \\
\hline 13 & 120 & 14 & & 180 & 15 \\
14 & 120 & 110 & & 180 & 15 \\
15 & 120 & 278 & & 180 & 56 \\
16 & 130 & 3 & & 180 & 30 \\
17 & 130 & 27 & 180 & 56 \\
18 & 130 & 219 & & 180 & 30 \\
19 & 150 & 1 & & 180 & 56 \\
20 & 150 & 7 & & 180 & 30 \\
21 & 150 & 55 & 180 & 15 \\
22 & 180 & 0.1 & 180 & 30 \\
23 & 180 & 1 & 180 & 15 \\
24 & 180 & 8 & 180 & 56 \\
\hline
\end{tabular}

\section{NIR measurements}

NIR reflectance spectra were measured before and after thermal/hygrothermal treatment from the radial face of the air-dried wood samples using a Fourier transform (FT)NIR spectrophotometer (Bruker, MATRIX-F; TE-InGaAs detector with a fiber optic probe) under laboratory conditions. The measurement area was $7 \mathrm{~mm}^{2}$, which covered more than 2 years of tree rings, allowing the averaged spectral information of early and late wood to be acquired. A white plate (barium sulfate) served as the reference signal. To improve the signal-to-noise ratio, 32 scans were co-added at a spectral resolution of $8 \mathrm{~cm}^{-1}$ over a wavenumber range of $10,000-4000 \mathrm{~cm}^{-1}$. A zero-filling of two (corresponding to a spectral interval of $4 \mathrm{~cm}^{-1}$ ) was applied. Three NIR spectral measurements were obtained and averaged for each sample.

\section{PCA and kinetic analysis}

Matlab (MathWorks, Inc., MA, USA) was used for spectral data pre-processing, calculation of the PCA score, and kinetic analysis. The NIR spectra were processed using 21 segments and a 0 gap to obtain second derivatives (gapsegment second derivatives). Although PCA of the NIR spectra of wood is generally conducted after mean-centering of the spectral data, we subtracted the averaged NIR second-derivative spectra of control wood samples (i.e., the wood samples before the thermal/hygrothermal treatment) from the NIR second-derivative spectra of the thermally/ hygrothermally treated wood samples before PCA analysis to allow selective evaluation, as explained in detail later.

\section{Results and discussion}

\section{NIR second-derivative spectra}

In the NIR spectra of wood, there is a spectral window between 6300 and $5450 \mathrm{~cm}^{-1}$ that lies between the two strong absorption bands of water at 7000 and $5200 \mathrm{~cm}^{-1}$ and contains the $\mathrm{C}-\mathrm{H}$ overtone bands. This spectral region is useful for the analysis of wood spectra because the effect of moisture content, which changes with species, heat treatment, and relative humidity, is lowest.

Figure 1a, d shows the second-derivative NIR spectra for the control wood samples (averaged across all samples prior to treatment), and wood samples that were thermally treated at $120{ }^{\circ} \mathrm{C}$ for 1152 and $6144 \mathrm{~h}$ for hinoki and zelkova, respectively, while Fig. 1b, e shows the second-derivative NIR spectra for the control wood samples, and wood samples that were hygrothermally treated at $120^{\circ} \mathrm{C}$ for 220 and $878 \mathrm{~h}$ for hinoki and zelkova, respectively.

The absorption band characteristics of the wood samples are labeled and their assignments [5] are summarized in Table 3. The absorption bands in the NIR region were conclusively associated with three polymers in wood: cellulose, hemicellulose, and lignin. In second-derivative spectra, lower absolute amplitudes of second-derivative values correspond with lower chemical concentrations. Peaks 2 and 5 were observed at the same wavelength in the control hinoki and zelkova samples, whereas peak 3 at $5867 \mathrm{~cm}^{-1}$ was only found in hinoki and peak 4 at $5809 \mathrm{~cm}^{-1}$ was only found in zelkova (Fig. 1a, d).

A different trend in spectral change with treatment time was observed for the thermal and hygrothermal treatments. For example, in hinoki, there was a clear increase and peak shift toward higher wavenumbers for the absorption band at $5983 \mathrm{~cm}^{-1}$ (peak 2 in Fig. 1a) following thermal treatment, which was attributed to $\mathrm{C}-\mathrm{H}$ vibrations in the aromatic rings/C-H groups in hemicellulose and extractives, 

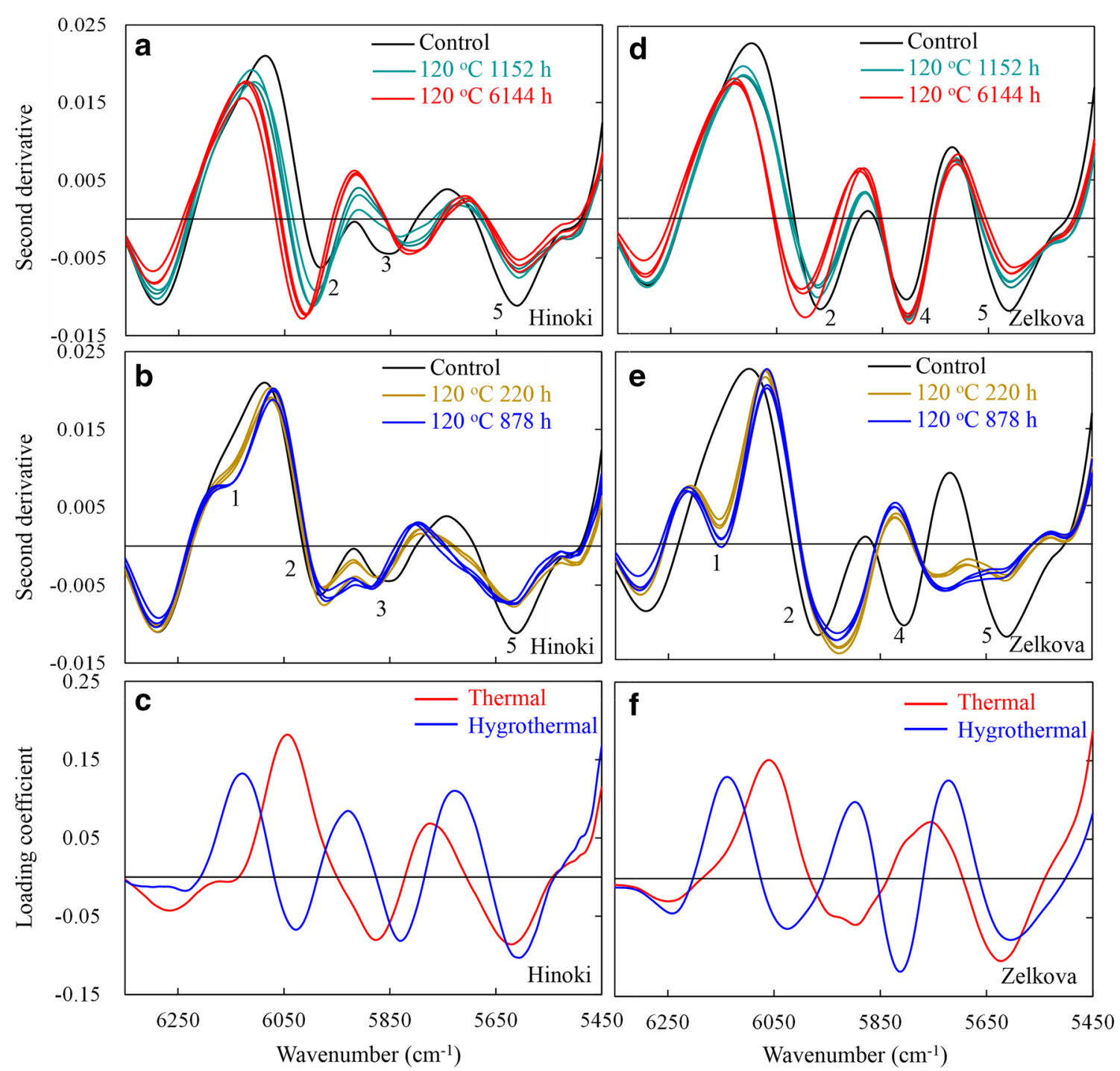

Fig. 1 NIR second derivative spectra $\left(6300-5450 \mathrm{~cm}^{-1}\right)$ of thermally treated wood, (a) hinoki-thermal, (b) hinoki-hygrothermal, (d) zelkovathermal, (e) zelkova-hygrothermal and loading coefficients of PC1 for (c) hinoki and (f) zelkova calculated from the PCA

whereas there was a peak shift toward lower wavenumbers following hygrothermal treatment (Fig. 1b). By contrast, the absorption band at $5867 \mathrm{~cm}^{-1}$ (peak 3 in Fig. 1a), which was assigned to the first overtone of $\mathrm{C}-\mathrm{H}$ vibrations in lignin, hemicellulose, and cellulose, showed a peak shift toward a lower wavenumber following thermal treatment and a peak shift toward a higher wavenumber following hygrothermal treatment (Fig. 1b). Finally, the absorption band at $5610 \mathrm{~cm}^{-1}$ (peak 5 in Fig. 1a) exhibited a decrease and a peak shift toward a lower wavenumber following thermal treatment and a peak shift toward a higher wavenumber following hygrothermal treatment (Fig. 1b). New peaks (peak 1 in Fig. 1b) only appeared following hygrothermal treatment. These spectral changes with heat treatment were well corresponding to previous reports $[4,6]$.
A comparison of the spectral changes in hinoki and zelkova showed that the two species exhibited different trends with treatment time, which could be explained by two differences between these species and between softwoods and hardwoods in general: (1) differences in their chemical structures and concentrations, i.e., the amplitudes of peaks 2 and 3 in the hinoki control samples were relatively small compared with peak 5 , whereas peaks 2 , 4 , and 5 had almost identical amplitudes in zelkova; and (2) differences in their resistance to heat treatment-it is well known that hardwood species generally experience greater weight loss following heat treatment than softwood species [1]. 
Table 3 Band assignments in NIR spectra of wood

\begin{tabular}{|c|c|c|c|c|}
\hline & Wave number $\left(\mathrm{cm}^{-1}\right)$ & Assignment & Component & $\begin{array}{l}\text { Wave number from } \\
\text { reference }\left(\mathrm{cm}^{-1}\right)\end{array}$ \\
\hline 1 & 6148 & 1st OT O-H str & Cell & 6126 \\
\hline \multirow[t]{6}{*}{2} & $5983-5967$ & 1st OT C-H str & Hemi & 6003 \\
\hline & & 1 st OT C $\mathrm{arm}-\mathrm{H}$ str & Extr & 5995 \\
\hline & & & Lig & 5980 \\
\hline & & & & 5974 \\
\hline & & & & 5963 \\
\hline & & & & 5978 \\
\hline \multirow[t]{4}{*}{3} & 5867 & 1st OT C-H str & - & 5900 \\
\hline & & & Lig & 5890 \\
\hline & & & Cell & 5872 \\
\hline & & & Hemi & 5865 \\
\hline \multirow[t]{3}{*}{4} & 5809 & 1st OT C-H str & Lig./Hemi./Cell & 5814,5816 \\
\hline & & & Hemi & 5800 \\
\hline & & & Lig & 5795 \\
\hline 5 & 5610 & 1 st OT C- $\mathrm{H}_{2}$ str & Cell & 5618 \\
\hline
\end{tabular}

OT overtone, Lig. lignin, Hemi. hemicellulose, Cell. cellulose, Extr. extractives

\section{Kinetic analysis of PCA scores}

To effectively evaluate the spectral changes with thermal/ hygrothermal treatment, we applied PCA analysis to the spectra of (1) thermally treated hinoki, (2) hygrothermally treated hinoki, (3) thermally treated zelkova, and (4) hygrothermally treated zelkova. For each analysis, the second-derivative spectra of wood samples within the $6300-5450 \mathrm{~cm}^{-1}$ range across all temperature and treatment times were subjected to PCA after subtracting the averaged second-derivative spectra of all control wood samples. The spectral shape of thermally/hygrothermally treated wood differed between hinoki and zelkova for each specific heat treatment time, but the first principal component (PC1) loading representing the direction in which variance in the spectral data was largest had a similar shape in both species (Fig. 1c, f). The explained variance for spectral variation by calculated PC1 score were 54\% (hinoki-thermal), $38 \%$ (hinoki-hygrothermal), 52\% (zelkova-thermal) and $61 \%$ (zelkova-hygrothermal), respectively.

Examination of the changes in the PC1 scores of the hinoki spectra as a function of the logarithmic treatment times showed that higher temperatures accelerated the change in the PC1 scores for both thermally and hygrothermally treated wood samples (Fig. 2a, d). We recently suggested that changes in PCA scores that are calculated from NIR spectra could be interpreted using an Arrhenius approach that involves the time-temperature superposition (TTSP) method [7], because the PCs represent the multiple reactions that are involved in the degradation of wood, the average weight of each of which can be considered to equate to the activation energy [4]. The temperature dependence of the degradation rate constant $(k)$ is described by the Arrhenius equation:

$k=A \exp \left(-\frac{E_{a}}{R T}\right)$,

where $A$ is a frequency factor, $E_{a}$ is the activation energy $(\mathrm{kJ}$ $\left.\mathrm{mol}^{-1}\right), R$ is the gas constant $\left(\mathrm{kJ} \mathrm{mol}^{-1} \mathrm{~K}^{-1}\right)$, and $T$ is the absolute temperature $(\mathrm{K})$. Thus, the activation energy can be calculated by determining the degradation rate.

TTSP is a reliable method for probing for Arrhenius behavior and considers time and temperature to be equivalent [7]. The parameters that are measured as a function of heat treatment time at different temperatures can be superimposed using an appropriate change in scale (referred to as the time-temperature shift factor, $a_{T}$ ) along the time axis, which is defined as:

$a_{T}=\frac{t_{\text {ref }}}{t_{T}}$,

where $t_{\text {ref }}$ is the heat treatment time at a reference temperature $T_{\text {ref }}$ and $t_{T}$ is the time that gives the same response at temperature $T$. Combining Eqs. (1) and (2) gives:

$a_{T}=\exp \left[\frac{E_{a}}{R}\left(\frac{1}{T_{\text {ref }}}-\frac{1}{T}\right)\right]$.

Equation (3) shows that the logarithm of the empirically determined shift factor $a_{T}$ has a linear relationship with the reciprocal absolute temperature $(T)$, which enables $E_{a}$ to be calculated and the shift factor to be predicted for a given temperature. Here we first used the data obtained at $180{ }^{\circ} \mathrm{C}$ as reference data to determine the master curve 

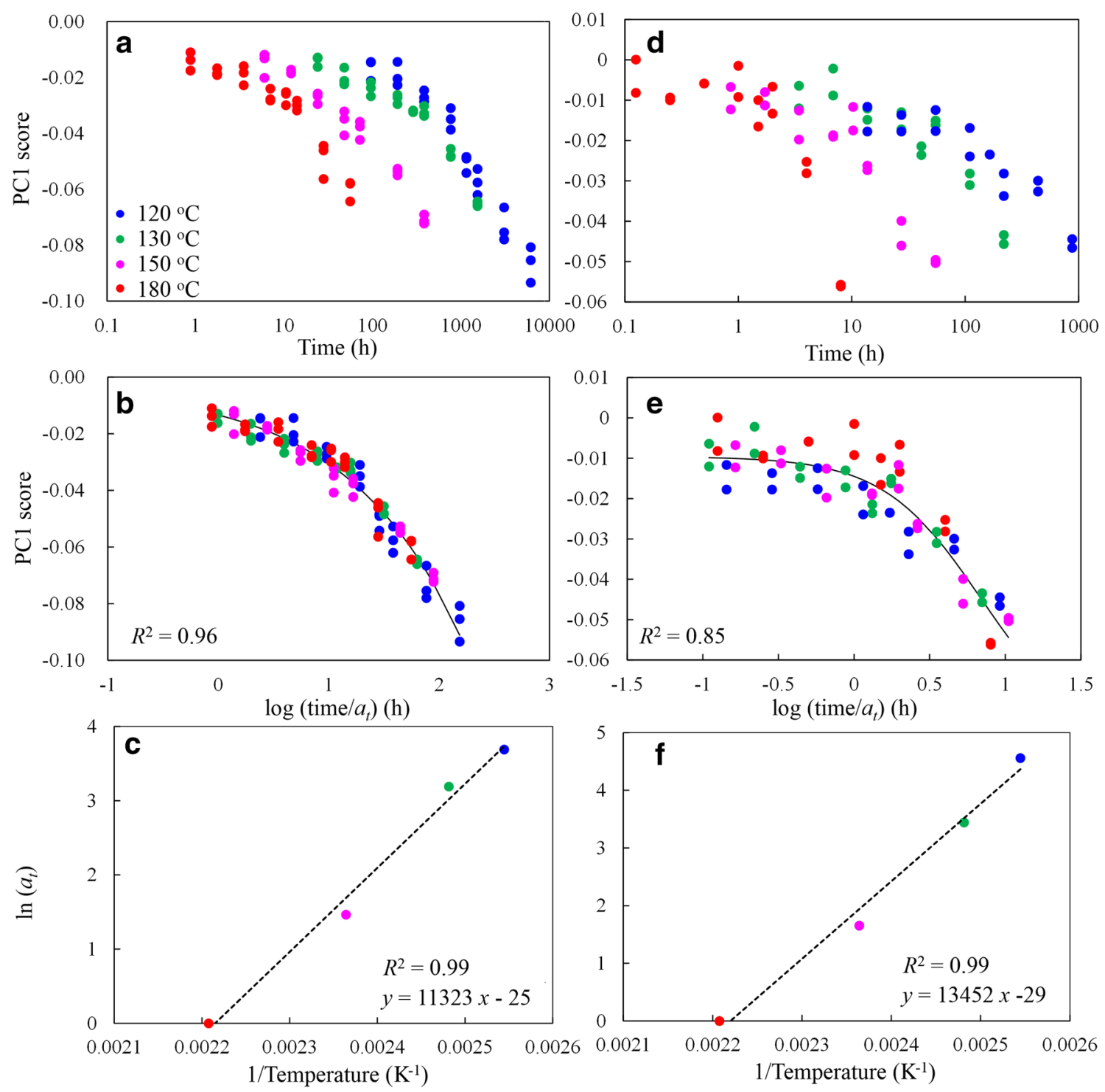

Fig. 2 Changes in the (a, d) PC1 score and (b, e) superimposed PC1 score calculated from NIR second derivative spectra (6300$5450 \mathrm{~cm}^{-1}$ ) as a function of treatment time. Blue circle, green circle, pink circle and red circle show the score of wood samples treated at $120,130,150$ and $180{ }^{\circ} \mathrm{C}$, respectively. $R^{2}$ shows the determination

across the entire temperature range, as the PCA score exhibited a large change with treatment time at this temperature. The PCA score was estimated using the following equation, which uses a nonlinear curve-fitting method, i.e., the simplex method, with a logistic function that adds the constant $\delta$ as an evaluation function:

$f(x)=\delta+\frac{\alpha}{1+\beta \exp (-\gamma x)}$,

where $f(x)$ is the PCA score, $x$ is $\log \left(t_{T} / a_{T}\right)$, and $\alpha, \beta$, $\gamma$, and $\delta$ are constants. coefficient between the measured and simulated values. (c, f) Arrhenius plot of shift factors from empirical superposition of the change of $\ln \left(a_{T}\right)$. Left figures show the result for thermal treatment and right figure shows the result for hygrothermal treatment. Species is hinoki

Once the master equation $(\alpha, \beta, \gamma$, and $\delta$ ) had been determined at the experimental temperature of $180{ }^{\circ} \mathrm{C}$ $\left(a_{180}{ }^{\circ} \mathrm{C}=1\right)$, the shift factor was determined, which was chosen empirically to produce the best overall superposition with the $180{ }^{\circ} \mathrm{C}$ data at each temperature $(120,130$, and $150{ }^{\circ} \mathrm{C}$ ). This then allowed the master curve to be applied to the data for each temperature. The superimposed PC1 score data with the regression curve are shown in Fig. 2b, e. The determination coefficients $\left(R^{2}\right)$ between the measured and simulated values and the root mean square errors (RMSE) of the superimposed curves were 0.96 and 
$4.0 \times 10^{-3}$, respectively, for the thermal treatment, and 0.83 and $5.5 \times 10^{-3}$, respectively, for the hygrothermal treatment, where $R^{2}$ and RMSE are defined as:

$R^{2}=1-\frac{\sum\left(y-y_{\text {pred }}\right)^{2}}{\sum(y-\bar{y})^{2}}$,

$\operatorname{RMSE}=\sqrt{\frac{\sum\left(y-y_{\text {pred }}\right)^{2}}{n-1}}$, where $y$ is the PCA score value, $\bar{y}$ is the average of $y, y_{\text {pred }}$ is the $y$ value predicted using the TTSP method, and $n$ is the number of $y$. These high $R^{2}$ values imply that the change in $\mathrm{PC} 1$ was caused by the same process independent of the thermal/hygrothermal treatment temperature, while the lower $R^{2}$ value for the hygrothermal treatment could be attributed to it having a shorter treatment time than the thermal treatment (0.1-8 vs. $0.875-56 \mathrm{~h}$, respectively, at $180{ }^{\circ} \mathrm{C}$; Table 1$)$. This resulted from the need to place the high-pressure cracking reactor containing wood samples and a piece of wet paper into an electric oven for the hygrothermal treatments because
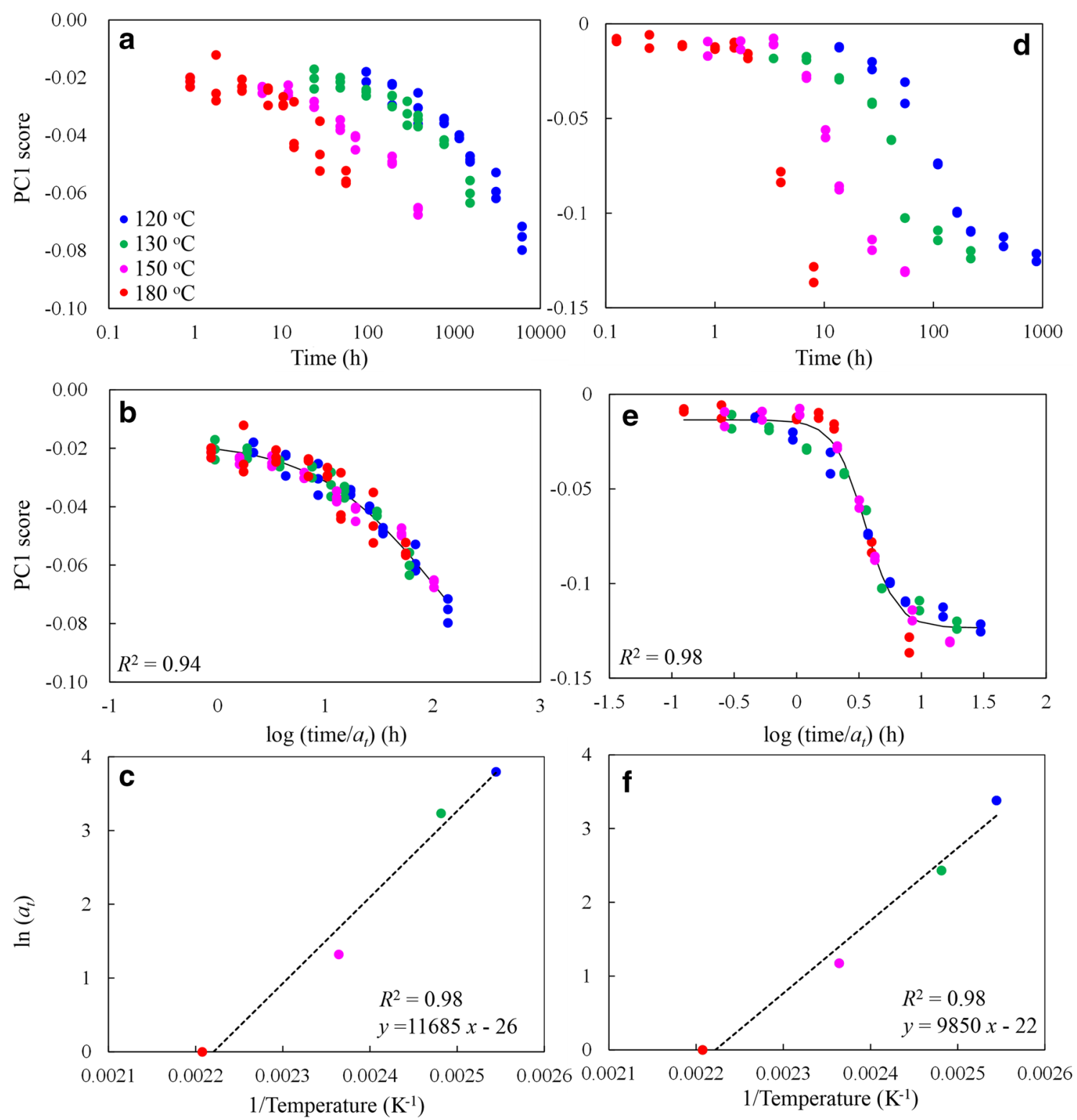

Fig. 3 Changes in the (a, d) PC1 score and (b, e) superimposed PC1 score calculated from NIR second derivative spectra (6300$5450 \mathrm{~cm}^{-1}$ ) as a function of treatment time. Blue circle, green circle, pink circle and red circle show the score of wood samples treated at $120,130,150$ and $180{ }^{\circ} \mathrm{C}$, respectively. $R^{2}$ shows the determination coefficient between the measured and simulated values. (c, f) Arrhenius plot of shift factors from empirical superposition of the change of $\ln \left(a_{T}\right)$. Left figures show the result for thermal treatment and right figure shows the result for hygrothermal treatment. Species is zelkova 
it took too long for the temperature inside the reactor to reach the required temperature, resulting in undesirable variation in the spectra of wood. As PC1 gave the best $R^{2}$ values for all cases (although the same approach were done for PC2, PC3, PC4, and PC5 for thermal and hygrothemal treatment), we consider that $\mathrm{PC} 1$ is reflecting the spectral change with heat treatment the most in all PC and decided to show the result only for $\mathrm{PC} 1$ data.

The relationship between $\ln \left(a_{T}\right)$ and $1 / T$ is shown in the Arrhenius plots provided in Fig. 2c, f. The linear relationship between these parameters implies that it is possible to predict the PC1 score in naturally aged wood by extrapolating the linear regression line to the appropriate temperature, e.g., $15^{\circ} \mathrm{C}$ to equate to the annual mean air temperature that is experienced in historical buildings in Nara, Japan. The PCA scores of the zelkova samples exhibited a similar trend to those of hinoki (Fig. 3), although the PC1 score appeared to be approaching equilibrium with the hygrothermal treatment (Fig. 3d, e).

\section{Selective assessment of duplex heat-treated wood}

Heat treatment is sometimes used to simulate the natural aging process in wood or paper [8]. For example, Matsuo et al. [9-12] subjected wood to temperatures of $90-180{ }^{\circ} \mathrm{C}$ under dry conditions accompanied by thermal oxidation to simulate archaeological wood. When they compared the color of aging wood samples from historical buildings with that of their heat-treated wood samples [10], they found that the real archaeological wood had a lower $a_{T}$ value for color properties than was calculated from the extrapolated value for the heat-treated samples. They argued that this could have been due to the non-linearity of the relationship between $\ln \left(a_{T}\right)$ and $1 / T$ over the reaction temperature range they used (i.e., $90-180{ }^{\circ} \mathrm{C}$ ) or the fact that naturally aging samples may exhibit an accelerated change due to moisture in the cell wall inducing hydrolysis in addition to oxidation during repetitive drying and wetting cycles, and hot and cold periods. This highlights the importance of being able to selectively assess the degree of oxidation and hydrolysis from the NIR spectra of duplex heat-treated wood.

To investigate whether such selective assessment is possible, we prepared 24 kinds of duplex heat-treated wood samples, as summarized in Table 2. To create these samples, thermally treated samples were exposed to an additional hygrothermal treatment and hygrothermally treated samples were exposed to an additional thermal treatment. These additional treatments were employed using only a temperature of $180{ }^{\circ} \mathrm{C}$ because it was found that the master curve that was constructed from the $180{ }^{\circ} \mathrm{C}$ data could be applied to the data from all other temperatures using the shift procedure, as shown in Fig. 2b, e.
Figure 4 shows the spectral space produced from 235 orthogonal coordinate axes (i.e., a wavenumber range of $6350-5450 \mathrm{~cm}^{-1}$ with $4 \mathrm{~cm}^{-1}$ steps). In this spectral space, each second-derivative spectrum is represented by a single dot. Because we subtracted the averaged second-derivative spectra of all control wood samples (before treatment) from the spectra of thermally/hygrothermally treated wood samples, the direction of the PC1 loading represents the direction of the spectral change with thermal/hygrothermal treatment. The spectra of duplex heat-treated wood samples should lie on the plane surface that is formed by the PC1 loadings of the thermal and hygrothermal treatments, as indicated by the green dots in Fig. 4. The angles between the PC1 loadings of the thermal and hygrothermal treatments, as calculated from the inner products, were $79^{\circ}$ for hinoki and $80^{\circ}$ for zelkova. Therefore, since these angles were close to orthogonal, it may be possible to selectively assess the thermal and hygrothermal treatment times for duplex heat-treated wood by calculating the inner product of the duplex heat-treated wood spectra after subtracting the spectra of control wood samples and the loading vectors for the thermal and hygrothermal treatment times.

The calculated scores as a function of $\log \left(t_{T} / a_{T}\right)$ are shown in Fig. 5. In this figure, the black filled circles show the PC1 scores for single thermally-treated (Fig. 5a, c) and hygrothermally-treated (Fig. 5b, d) wood samples, while the black solid lines represent the master curves. The green circles show the scores that were calculated from the inner

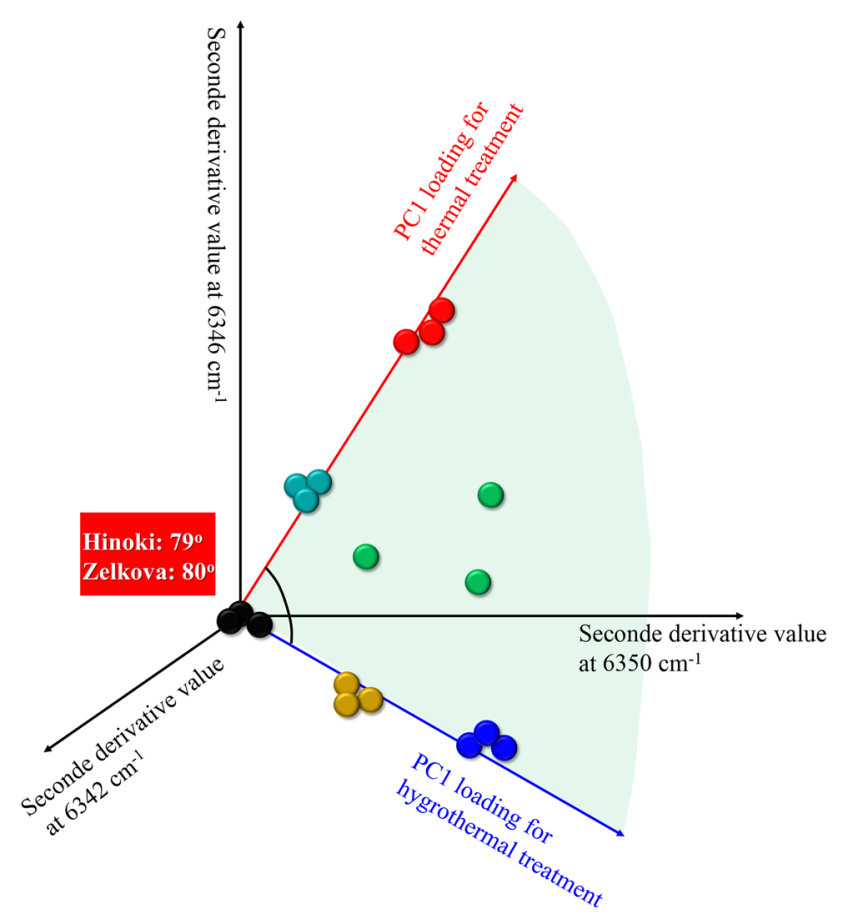

Fig. 4 Image of spectral space having 235 orthogonal coordinate axis 

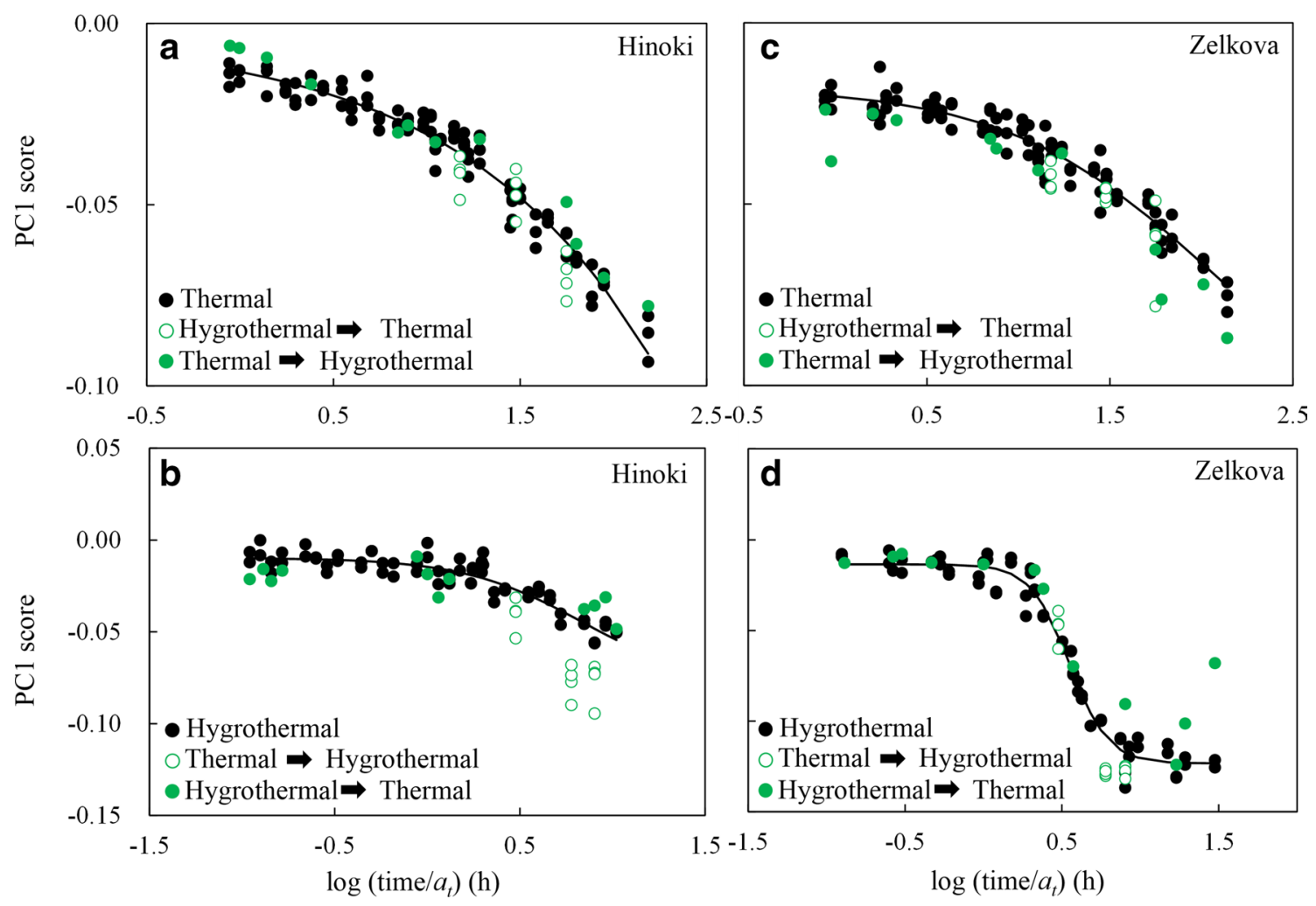

Fig. 5 Calculated score as a function of $\log \left(t_{T} / a_{T}\right)$. Black filled circle shows the change of PC1 score for single treated wood (a, $\mathbf{c}$ thermal treatment, b, d hygrothermal treatment). Black solid line shows the master curve. Green circle shows the score calculated by the inner product of duplex heat-treated wood spectra with loading vector for thermal treatment and hygrothermal treatment (a, $\mathbf{c}$ Green opened circle show the inner product score of spectra of hygrothermally treated wood followed by the thermal treatment with loading of thermal

product of duplex heat-treated wood spectra using the loading vectors for the thermal and hygrothermal treatments. From these plots it can be seen that the thermal treatment times were well predicted from the NIR spectra of duplex thermally treated wood samples from both species (Fig. 5a, c), as the green circles sit along the master curve. The RMSE values of the duplex-treated wood samples from the master curve were approximately $2-3$ times bigger than the RMSE values for the single-treated wood samples for the thermal treatment but 1.6-5.5 times bigger than the single-treated wood samples for the hygrothermal treatment. These hygrothermal RMSE values are too high to allow the accurate prediction of treatment times, largely due to the time the hygrothermal samples took to reach the desired temperature and the accompanying variation in the spectra of these samples, as explained previously, the method proposed in this study is useful for two reasons: (1) it allows the thermal history of wood to be determined by measuring only the NIR spectra; and (2) the master curve that was acquired in this study could be applied to any temperature using the predicted $a_{T}$ treatment, green closed circle show the inner product score of spectra of thermally treated wood followed by the hygrothermal treatment with loading of thermal treatment. $\mathbf{b}, \mathbf{d}$ Green opened circle show the inner product score of spectra of thermally treated wood followed by the hygrothermal treatment with loading of hygrothermal treatment, green closed circle show the inner product score of spectra of hygrothermally treated wood followed by the thermal treatment with loading of hygrothermal treatment.)

value from the Arrhenius relationship, as shown in Fig. 2c. These advantages imply that this method could be used to evaluate the thermal history of all kinds of natural aging wood samples. Future research that considers changes in the NIR spectra of wood that has been subjected to ultraviolet (UV) irradiation (light degradation) and fungus treatment would be useful to determine whether the loading vector that explains the NIR changes due to these factors is perpendicular to the loading vector for thermal or hygrothermal treatment.

\section{Conclusion}

The master curve that we calculated by kinetic analysis successfully explained the changes in the PC1 scores with thermal and hygrothermal treatment time across all temperatures. By calculating the inner product between the secondderivative spectra of duplex heat-treated wood samples and the loading vector that explains the spectral variation due to 
thermal or hygrothermal treatment, we were able to selectively assess the thermal and hygrothermal treatment times. Although the RMSE values for the duplex heat-treated wood samples were too high for accurate assessment, we demonstrate that this is a useful method for assessing the thermal history of wood.

Acknowledgements The authors would like to acknowledge the financial support from JSPS (KAKENHI, No. 26850111).

\section{References}

1. Esteves B, Pereira HM (2009) Wood modification by heat treatment: a review. Bioresources 4:370-404

2. Esteves B, Pereira HM (2008) Quality assessment of heat-treatedwood by NIR spectroscopy. Holz Roh Werkst 66:23-332

3. Kobori H, Tsuchikawa S (2015) A review of recent application of near infrared spectroscopy to wood science and technology. J Wood Sci 61:213-220

4. Inagaki T, Matsuo M, Tsuchikawa S (2016) NIR spectral-kinetic analysis for thermally degraded sugi (Cryptomeria japonica) wood. Appl Phys A 122:208

5. Schwanninger M, Rodrigues JC, Fackler K (2011) A review of band assignments in near infrared spectra of wood and wood components. J Near Infrared Spec 19:287-308
6. Inagaki T, Mitsui K, Tsuchikawa S (2008) Near-infrared spectroscopic investigation of the hydrothermal degradation mechanism of wood as an analogue of archaeological objects. Part I: softwood. Appl Spectrosc 62:1209-1215

7. Ding HZ, Wang ZD (2007) Time-temperature superposition method for predicting the permanence of paper by extrapolating accelerated ageing data to ambient conditions. Cellulose 14:171-181

8. Zou X, Uesaka T, Gurnagul N (1996) Prediction of paper permanence by accelerated predictions aging II. Comparison of the predictions with natural aging results. Cellulose 3:269-279

9. Matsuo M, Yokoyama M, Umemura K, Gril J, Yano K, Kawai S (2010) Color changes in wood during heating: kinetic analysis by applying a time-temperature superposition method. Appl Phys A 99:47-52

10. Matsuo M, Yokoyama M, Umemura K, Sugiyama J, Kawai S, Gril J, Kubodera S, Mitsutani T, Ozaki H, Sakamoto M, Imamura M (2011) Aging of wood: analysis of color changes during natural aging and heat treatment. Holzforschung 65:361-368

11. Matsuo M, Umemura K, Kawai S (2012) Kinetic analysis of color changes in cellulose during heat treatment. J Wood Sci 58:113-119

12. Matsuo M, Umemura K, Kawai S (2014) Kinetic analysis of color changes in keyaki (Zelkova serrata) and sugi (Cryptomeria japonica) wood during heat treatment. J Wood Sci 60:12-20 